\title{
Prognostic predictors of remission in ocular myasthenia after thymectomy
}

\author{
Xiang Liu ${ }^{1}$, Wangyan Zhou ${ }^{2}$, Jun $\mathrm{Hu}^{1}$, Mingsong Hu${ }^{1}$, Wenkui Gao ${ }^{1}$, Shan Zhang ${ }^{1}$, Wei Zeng ${ }^{1}$ \\ ${ }^{1}$ Department of Cardiothoracic Surgery, the Second Affiliated Hospital, University of South China, Hengyang 421001, China; ${ }^{2}$ Department of \\ Medical Humanities and Education Department, the First Affiliated Hospital, University of South China, Hengyang 421001, China \\ Contributions: (I) Conception and design: X Liu, W Zeng; (II) Administrative support: All authors; (III) Provision of study materials or patients: \\ X Liu, W Zhou, J Hu, M Hu, W Gao, S Zhang; (IV) Collection and assembly of data: X Liu, W Zhou, J Hu, M Hu, W Gao, S Zhang; (V) Data \\ analysis and interpretation: All authors; (VI) Manuscript writing: All authors; (VII) Final approval of manuscript: All authors. \\ Correspondence to: Wei Zeng. Department of Cardiothoracic Surgery, the Second Affiliated Hospital, University of South China, Jiefang Avenue 35, \\ Hengyang 421001, China. Email: drmedzengwei@gmail.com.
}

Background: Identifying prognostic predictors can assist in making clinical decisions. This study aimed to identify the potential predictors of remission in patients with ocular myasthenia gravis (OMG) after thymectomy.

Methods: OMG patients who had thymectomy between 2011 and 2017 were reviewed retrospectively. Clinical outcomes were assessed according to the Myasthenia Gravis Foundation of America PostIntervention Status (MGFA-PIS). Kaplan-Meier analysis was used to estimate the cumulative probability of complete stable remission (CSR). Univariate and multivariable analyses with Cox proportional hazards regression were used to identify predictors of CSR.

Results: Fifty-one patients (23 male, 28 female) with a median age at OMG onset of 40 (range, 5-79) years were eligible for inclusion. Patients with thymomatous OMG (n=9) had a statistically older median age at disease onset [61 (range, 32-78) vs. 33.5 (range, 5-79) years, $\mathrm{P}=0.001$ ], shorter duration from disease onset to thymectomy [3 (range, 2-24) vs. 10 (range, 1-132) months, $\mathrm{P}=0.004$ ], and a higher rate of postoperative complication $(44.4 \%$ vs. $9.5 \%, \mathrm{P}=0.025)$, compared with non-thymomatous $\mathrm{OMG}(\mathrm{n}=42)$. The estimated cumulative probability of CSR in the whole cohort was $41.8 \%$ (95\% CI, 28.6-58.2\%) 5 years after surgery. Age at onset of 40 years or younger $(\mathrm{P}=0.00016)$, female sex $(\mathrm{P}=0.069)$, and thymic hyperplasia $(\mathrm{P}=0.0061)$ were potential predictors under univariate analysis. However, only age at onset of 40 years or younger (HR: 4.117, 95\% CI, 1.177-14.399, P=0.027) remained significant after multivariable analysis.

Conclusions: CSR could be achieved in about $40 \%$ of OMG patients 5 years after thymectomy and is likely to be predicted by age at onset of 40 years or younger.

Keywords: Ocular myasthenia gravis (OMG); thymectomy; predictor; clinical outcome

Submitted Oct 29, 2019. Accepted for publication Dec 30, 2019.

doi: $10.21037 /$ jtd.2020.01.17

View this article at: http://dx.doi.org/10.21037/jtd.2020.01.17

\section{Introduction}

Thymectomy has become a treatment option in multidisciplinary myasthenia gravis (MG) management since Blalock first reported a marked improvement in $\mathrm{MG}$ in a 21-year-old woman following the removal of a cystic thymic tumor in 1939 (1). Despite this early success, it was not until 2016 that the Myasthenia Gravis Thymectomy Trial (MGTX) could finally determine the therapeutic value of thymectomy for generalized MG (2). In fact, the 5 -year findings of MGTX further confirmed the continuing advantage of thymectomy in strengthening symptoms and reducing prednisone use in patients with generalized MG (3). However, the role of thymectomy in ocular MG 
currently remains controversial.

At disease onset, about half of MG patients present with purely ocular symptoms, typically ptosis and/or diplopia, of whom more than half will develop a secondary generalization, mostly within 2 years (1-3). Ocular myasthenia gravis (OMG), which is commonly considered a "mild" disease, can induce profound visually disability, and, in some cases, can potentially impair quality of life (4). A national investigation from the United States observed 804,674 ambulatory and 49,790 emergency department visits for diplopia annually between 2003 and 2012 (5). With the advent of the thoracoscope, minimally invasive thymectomy has surpassed large incisions as an option which promises lower postoperative morbidity and mortality as well as faster recovery, resulting in an emerging acceptance of thymectomy in patients with OMG. A metaanalysis of retrospective studies has demonstrated that thymectomy seems to be safe and effective for patients with OMG in terms of inducing remission (6). However, it is likely that thymectomy cannot benefit each patient with OMG. Identification of predictors of remission after thymectomy may help optimize patient selection for surgery and avoid exposing patients to related therapeutic risks. In this retrospective study, we aimed to identify predictive factors of remission in OMG patients undergoing thymectomy.

\section{Methods}

\section{Study design}

This was a retrospective cohort study. Ethics approval was granted by the ethics committee and institutional review board of our institution. Written informed consent of surgery and the use of clinical data were obtained from all patients involved in this study.

\section{Patient selection}

Patients with a confirmed diagnosis of OMG who underwent thymectomy between 2011 and 2017 were retrospectively reviewed for inclusion. Preoperative characteristics and surgical data were collected from the hospital information system.

The diagnosis and workup of OMG can be as vexing as formulating a treatment plan. Typically, the diagnosis is confirmed based on clinical symptoms (mostly ptosis and/or diplopia) and either positive auto-antibody status [mostly acetylcholine receptor antibody (anti-AChR)], positive neurophysiological tests (including single-fiber electromyography and repetitive nerve stimulation), or characteristic response to therapy $(4,7)$. OMG patients with thymoma are indicated for thymectomy. However, the application of thymectomy in patients with nonthymomatous OMG is still controversial. In general, we recommend that patients with OMG undergo a thymectomy if immunosuppressive medications are not able to control the symptoms, or if there is a need to avoid or reduce the use of immunosuppressive medications. However, patients were asked to make their own decision of surgery after a comprehensive explanation of the pros and cons of both medical treatment and surgical treatment.

\section{Surgical procedure}

Thymectomy was performed through the right-sided videoassisted thoracoscopic surgery (VATS) approach. In this approach, each patient is placed in supine position with about a 30-degree elevation of the right hemithorax. This position allows easy access to the anterior mediastinum and convenient conversion to both the sternotomy and thoracotomy approaches. The thoracoscope incision is in the fourth intercostal space at the midaxillary line for the insertion of a 30-degree telescope. The other 2 incisions are in the third intercostal space at the midaxillary line and in the sixth intercostal space at the anterior axillary line, respectively. In this study, dissection started from the right inferior horn of the thymus and extended cranially along the right phrenic nerve. The thymic veins were managed by ultrasonic or electrocautery dissection, and the superior horns were dissected after gentle and careful retraction. In general, both phrenic nerves should be observed, and all mediastinal fat should be dissected free. Specimens were removed in a retrieval bag.

\section{Follow-up}

In general, patients attended the outpatient department in our clinic for adjustment of MG medications every 3 to 6 months. Those who stopped visiting the clinic were followed up by a telephone interview. The clinical outcome was assessed according to the Myasthenia Gravis Foundation of America Post-Intervention Status (MGFAPIS). Complete stable remission (CSR) was defined as no 
Table 1 Preoperative characteristics of patients with OMG who underwent thymectomy

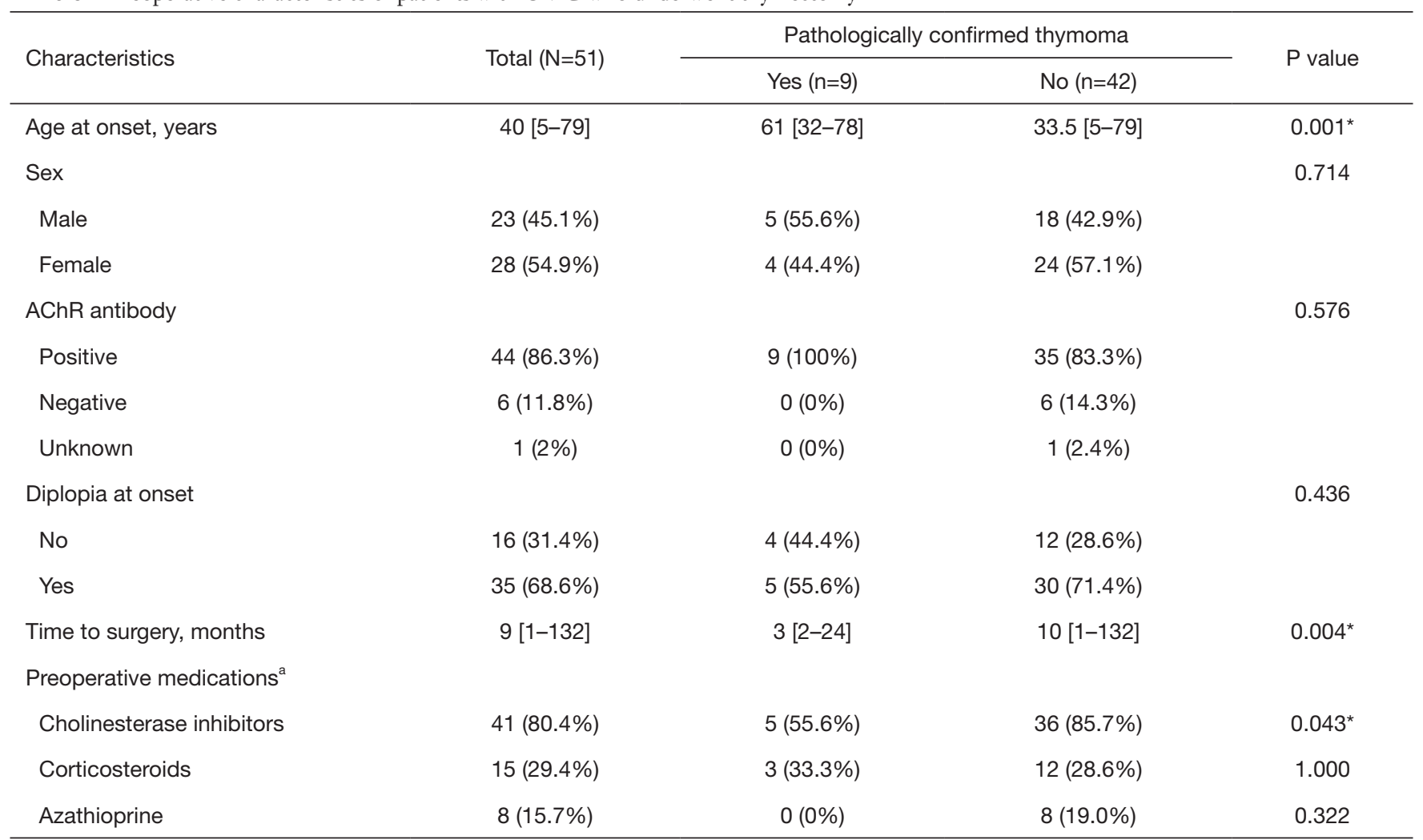

${ }^{a}$, data were available for 50 patients (9 thymomatous OMG versus 41 nonthymomatous OMG). * , statistically significant. OMG, ocular myasthenia gravis.

symptoms and without medications for at least 1 year.

\section{Statistical analysis}

SPSS 22.0 (IBM Corp., Armonk, NY, USA) and R statistical software version 3.5.3 were used to perform the statistical analyses. Patients were categorized into 2 groups according to the presence of a thymoma. Continuous variables and categorical variables were described as median (range) and number (proportion), respectively, because all continuous variables in this study were not normally distributed. Mann-Whitney test for continuous variables and Chisquare test or Fisher's exact test for categorized variables were used to compare preoperative characteristics and clinical outcomes between the 2 groups. Fisher's exact test was used when more than $20 \%$ of cells had expected frequencies $<5$. The Kaplan-Meier method was used to plot the curves of the cumulative probability of CSR, and a logrank test was used for comparison between subgroups. Also, we conducted univariate analyses with Cox proportional hazards regression to find potential predictors of CSR after thymectomy. Factors with a $\mathrm{P}$ value smaller than 0.1 were included for final multivariable analysis. A $\mathrm{P}$ value $<0.05$ was considered statistically significant.

\section{Results}

Between 2011 and 2017, 62 patients with OMG underwent thymectomy in our hospital. After excluding 11 (17.7\%) patients who were lost to follow-up, 51 patients (23 male, 28 female) were eligible for inclusion with a median age at disease onset of 40 (range, 5-79) years. Of these, 9 (17.6\%) patients had a pathologically confirmed thymoma, whereas $42(82.4 \%)$ patients did not. Table 1 shows the preoperative characteristics of the 51 patients with OMG. Patients with thymomatous OMG had a statistically older median age at disease onset [61 (range, 32-78) years] than those with nonthymomatous OMG [33.5 (range, 5-79) years, $\mathrm{P}=0.001]$. Furthermore, there was a significant difference in duration from disease onset to surgery between patients 
Table 2 Clinical outcomes of patients with OMG who underwent thymectomy

\begin{tabular}{|c|c|c|c|c|}
\hline Characteristics & Total $(\mathrm{N}=51)$ & \multicolumn{2}{|c|}{ Pathologically confirmed thymoma } & $P$ value \\
\hline Open conversion & $1(2.0 \%)$ & $1(11.1 \%)$ & $0(0 \%)$ & 0.176 \\
\hline Postoperative complications & $8(15.7 \%)$ & $4(44.4 \%)$ & $4(9.5 \%)$ & $0.025^{\star}$ \\
\hline Delirium & $1(2.0 \%)$ & $0(0 \%)$ & $1(2.4 \%)$ & \\
\hline Pneumothorax & $1(2.0 \%)$ & $0(0 \%)$ & $1(2.4 \%)$ & \\
\hline Pleural effusion & $2(3.9 \%)$ & $1(11.1 \%)$ & $1(2.4 \%)$ & \\
\hline Respiratory failure & $1(2.0 \%)$ & $1(11.1 \%)$ & $0(0 \%)$ & \\
\hline Myasthenia crisis & $2(3.9 \%)$ & $2(22.2 \%)$ & $0(0 \%)$ & \\
\hline Thymic hyperplasia & $13(25.5 \%)$ & - & $13(31.0 \%)$ & \\
\hline Thymoma & $9(17.6 \%)$ & $9(100 \%)$ & $0(0 \%)$ & \\
\hline Follow-up time, months & 45 [11-93] & 40 [20-93] & $47[11-93]$ & 1.000 \\
\hline Achievement of CSR & $20(39.2 \%)$ & $1(11.1 \%)$ & $19(45.2 \%)$ & 0.072 \\
\hline Secondary generalization & $8(15.7 \%)$ & $3(33.3 \%)$ & $5(11.9 \%)$ & 0.137 \\
\hline Change in status & & & & 0.625 \\
\hline Improved & $46(90.2 \%)$ & $8(88.9 \%)$ & 38 (90.5\%) & \\
\hline Unchanged & $3(5.9 \%)$ & $1(11.1 \%)$ & $2(4.8 \%)$ & \\
\hline
\end{tabular}

*, statistically significant. CSR, complete stable remission.

with thymomatous OMG [3 (range, 2-24) months] and patients with nonthymomatous OMG [10 (range, 1-132) months, $\mathrm{P}=0.004]$. In addition, we also found a difference in the preoperative use of cholinesterase inhibitors between the 2 groups $(\mathrm{P}=0.043)$. However, no differences were found as to sex, AChR antibody status, and symptoms at onset between the 2 groups.

The clinical outcomes of 51 patients with OMG after thymectomy are detailed in Table 2. In our series, 1 (2\%) patient with thymoma experienced conversion to open surgery due to an injury of the left brachiocephalic vein. The rate of postoperative complications in patients with thymomatous OMG was statistically higher than that in patients with nonthymomatous OMG (44.4\% vs. $9.5 \%$, $\mathrm{P}=0.025$ ), with a median follow-up time of 45 (range, 11-93) months. Although no statistical difference was found $(\mathrm{P}=0.137)$, there was a trend that patients with thymomatous OMG seemed to have a higher rate of secondary generalization $(33.3 \%)$, compared with patients with nonthymomatous OMG (11.9\%). Regarding the achievement of CSR, however, there was a marginal difference $(11.1 \%$ in thymomatous OMG vs. $45.2 \%$ in nonthymomatous OMG, $\mathrm{P}=0.072$ ) between the 2 groups. At the last follow-up, the majority $(88.9 \%)$ of patients with thymomatous OMG 
Strata + All
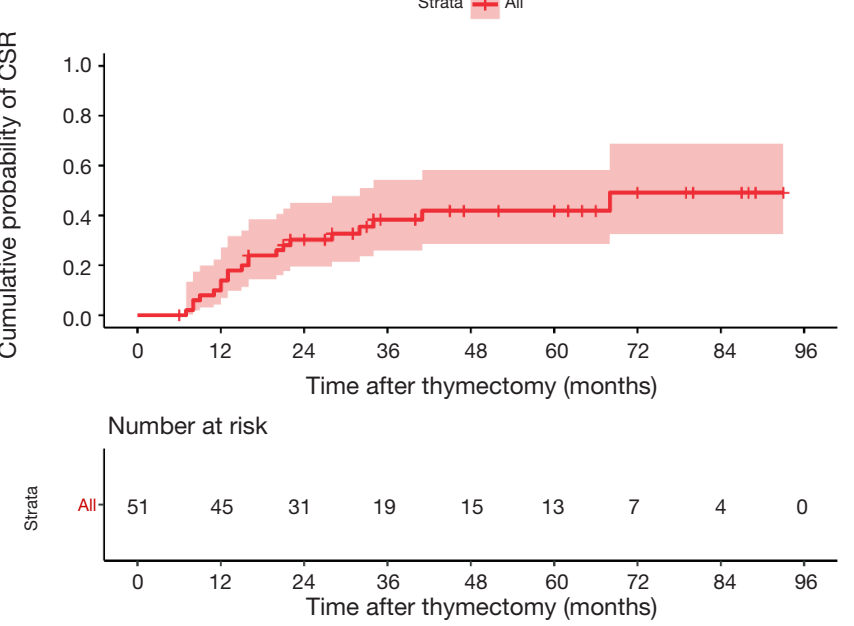

C

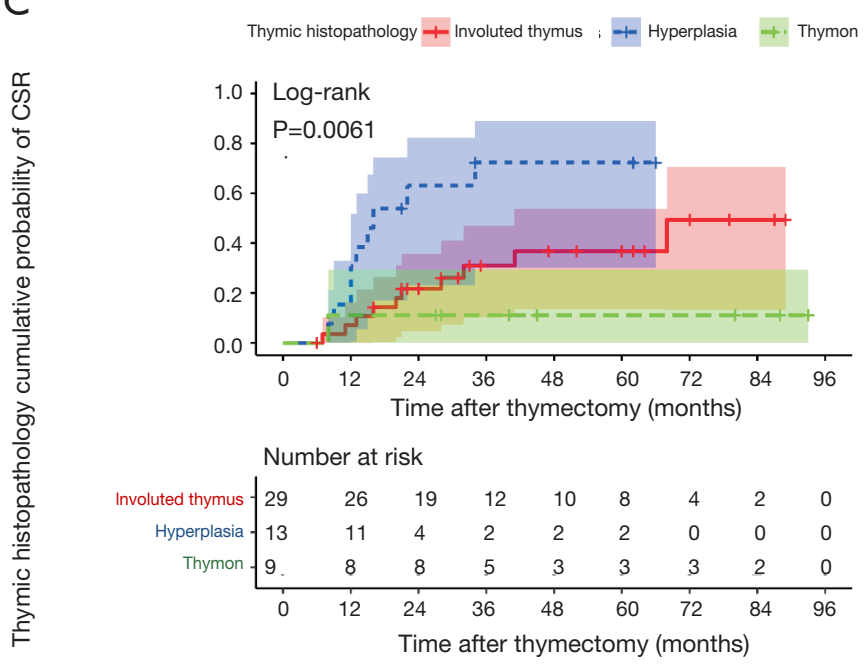

B

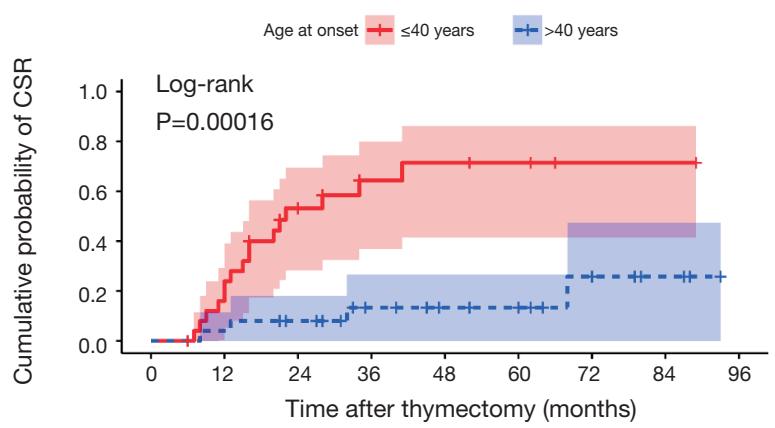

Number at risk

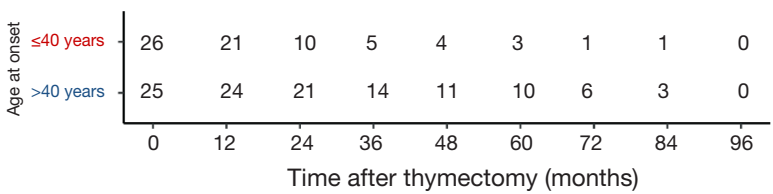

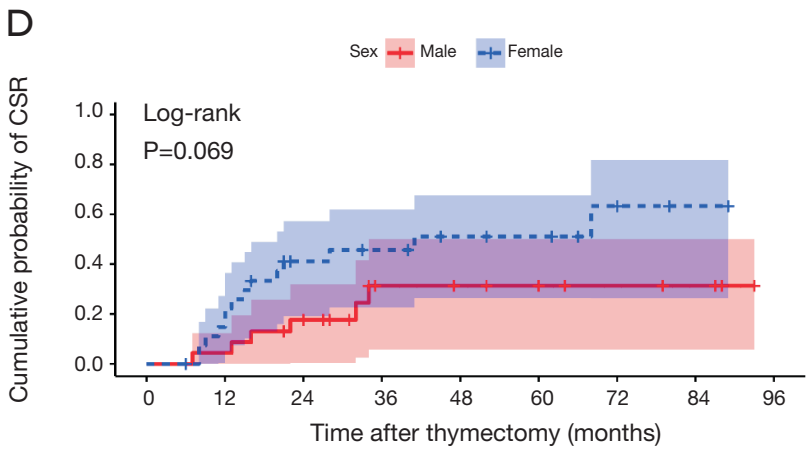

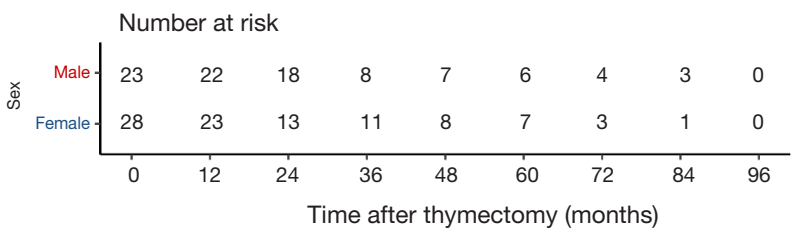

Figure 1 Kaplan-Meier curves for the cumulative probability of CSR. (A) Kaplan-Meier curves for the cumulative probability of CSR for the whole cohort. (B) Kaplan-Meier curves for the cumulative probability of CSR for the whole cohort stratified by age at disease onset. (C) Kaplan-Meier curves for the cumulative probability of CSR for the whole cohort stratified by thymus histopathology. (D) Kaplan-Meier curves for the cumulative probability of CSR for the whole cohort stratified by sex. CSR, complete stable remission.

required cholinesterase inhibitors, whereas only $18(42.9 \%)$ patients with nonthymomatous OMG did so $(\mathrm{P}=0.024)$.

Kaplan-Meier analysis was used to estimate the cumulative probability of CSR in patients with OMG after thymectomy. In the whole cohort, the estimated cumulative probability of CSR was $41.8 \%$ (95\% CI, 28.6-58.2\%) at 5 years (Figure $1 A$ ). Kaplan-Meier analysis demonstrated significant differences in the cumulative probabilities of CSR between subgroups stratified by age at disease onset (Figure $1 B, \mathrm{P}=0.00016$ ) and thymus histopathology (Figure $1 C, \mathrm{P}=0.0061$ ), and marginal differences between subgroups stratified by sex (Figure $1 D, \mathrm{P}=0.069)$. The estimated cumulative probabilities of CSR at 5 years were $71.5 \%$ (95\% CI, 41.4-86.1\%) for patients with an age at onset of 40 years or younger, 13.4\% (95\% CI, 0-26.6\%) for patients with an age at disease onset older than 40 years, $31.4 \%$ (95\% CI, 5.8-50\%) for male patients, and $51.1 \%$ (95\% CI, 26.3-67.6\%) for female patients. Regarding 
Table 3 Cox proportional hazards regression model analysis of clinical characteristics associated with remission after thymectomy

\begin{tabular}{|c|c|c|c|c|}
\hline Variable & \multicolumn{2}{|c|}{ Univariate analysis } & \multicolumn{2}{|c|}{ Multivariable analysis } \\
\hline Age at onset $<40$ years & $6.617(2.155-20.321)$ & $0.001^{*}$ & 4.117 (1.177-14.399) & $0.027^{\star}$ \\
\hline Female sex & $2.360(0.905-6.155)$ & $0.079^{*}$ & $1.577(0.587-4.242)$ & 0.367 \\
\hline Positive for AChR antibody & $1.516(0.349-6.582)$ & 0.579 & NA & NA \\
\hline TX within 1 year after onset & $0.741(0.307-1.790)$ & 0.506 & NA & NA \\
\hline Thymic hyperplasia $^{a}$ & $3.008(1.191-7.697)$ & $0.020^{*}$ & $1.720(0.655-4.521)$ & 0.271 \\
\hline Thymoma $^{a}$ & $0.280(0.036-2.193)$ & 0.225 & $0.512(0.060-4.388)$ & 0.541 \\
\hline
\end{tabular}

${ }^{a}$, reference was involuted thymus. *, statistically significant. HR, hazard ratio; CI, confidence interval; NA, not applicable; TX, thymectomy.

thymus histopathology, Kaplan-Meier analysis revealed the cumulative probabilities of CSR at 5 years of $36.8 \%$ (95\% CI, $13.4-53.8 \%$ ) for patients with involuted thymus, $72.3 \%$ (95\% CI, 30.1-89\%) for patients with thymic hyperplasia, and $11.1 \%$ (95\% CI, 0-29.4\%) for patients with thymoma. On the other hand, for patients with nonthymomatous OMG, the estimated cumulative probability of CSR at 5 years was $48.3 \%$ (95\% CI, 28.5-62.6\%).

Table 3 summarizes the Cox proportional hazards regression model analysis of the clinical characteristics associated with CSR after thymectomy. In univariate analyses, age at disease onset of 40 years or younger (HR: 6.617, 95\% CI, 2.155-20.321, $\mathrm{P}=0.001$ ), female sex (HR: 2.360, 95\% CI, 0.905-6.155, $\mathrm{P}=0.079$ ), and thymic hyperplasia (HR:3.008, 95\% CI, 1.191-7.697, $\mathrm{P}=0.020$ ) were identified as potential predictors of CSR after thymectomy (Table 3). In the final multivariable analysis, only age at disease onset of 40 years or younger (HR: 4.117, 95\% CI, 1.177-14.399, $\mathrm{P}=0.027$ ) persisted as an independent predictor of CSR after thymectomy (Table 3). Furthermore, subgroup analyses were performed in patients with non-thymomatous OMG, the results of which are summarized in Table 4. Similarly, only age at disease onset of 40 years or younger was associated with CSR after thymectomy in the final multivariable analysis.

\section{Discussion}

This retrospective cohort study aimed to define the CSR predictors in thymectomy patients with OMG. The perioperative mortality rate was $0 \%$ and the rate of morbidity was $15.7 \%$. The calculated average likelihood of CSR, with a mean follow-up time of 45 (range, 11-93) months, was $41.8 \%$ (95\% CI, 28.6-58.2\%) at 5 years after surgery. Age at disease onset of 40 years or younger was independently associated with the achievement of CSR after surgery.

OMG is not a life-threatening disease, but a confined disease with only ocular symptoms. Even so, diplopia may cause severe visual disability, potentially resulting in a low quality of life. Approximately 800,000 ambulatory and 50,000 emergency department visits for diplopia were observed annually in the United States (5). In our series, $35(68.6 \%)$ patients experienced diplopia at disease onset, which is comparable with that reported in another Chinese cohort study (8). Furthermore, previous studies have reported that about $90 \%$ of patients with OMG suffer from diplopia $(9,10)$.

Moreover, more than half of the patients with OMG can develop a secondary generalization, typically within 2 years (1-3). Previous studies have shed light on the efficacy of immunosuppressive medications on patients with OMG in terms of preventing secondary generalization $(11,12)$. However, as with any disease, treatment-related risks must be weighed against the potential benefits. In patients with OMG, even a lowto-moderate dose of corticosteroids can result in adverse effects in approximately $35 \%$ of patients (13). In light of this, several studies have tried to identify risk factors of secondary generalization to avoid unnecessary adverse effects caused by immunosuppressive medications $(9,10,14,15)$. Thus far, however, it has been challenging 
Table 4 Cox proportional hazards regression model analysis of clinical characteristics associated with remission after thymectomy in nonthymomatous patients

\begin{tabular}{|c|c|c|c|c|}
\hline Variable & \multicolumn{2}{|c|}{ Univariate analysis } & \multicolumn{2}{|c|}{ Multivariable analysis } \\
\hline Age at onset $\leq 40$ years & $6.530(1.865-22.858)$ & $0.003^{*}$ & 4.877 (1.264-18.822) & $0.021^{*}$ \\
\hline Female sex & $2.030(0.769-5.359)$ & 0.153 & $0.746(0.273-2.038)$ & 0.567 \\
\hline Positive for AChR antibody & $1.908(0.437-8.339)$ & 0.390 & NA & NA \\
\hline TX within 1 year after onset & $0.926(0.375-2.286)$ & 0.868 & NA & NA \\
\hline Thymic hyperplasia $^{a}$ & $3.132(1.232-7.959)$ & $0.016^{*}$ & $0.586(0.220-1.556)$ & 0.283 \\
\hline
\end{tabular}

${ }^{a}$, reference was involuted thymus. *, statistically significant. HR, hazard ratio; Cl, confidence interval; NA, not applicable; TX, thymectomy.

to stratify patients with OMG into subgroups with different risk levels of secondary generalization. Therefore, identifying the predictors of CSR after thymectomy might be beneficial and may allow us to better counsel patients about formulating a treatment plan.

Although very few studies have specifically investigated the role of thymectomy in OMG, a metaanalysis of 26 studies with a median eligible sample size of 16 (range, 3-105) showed that thymectomy is beneficial in terms of inducing remission in patients with OMG (9). Of these studies, one with a sample size of 61 patients demonstrated that thymectomy could lead to an apparent cure (symptoms free without medications) in $51 \%$ of patients with OMG (16). In 2011, a case series of 110 patients with OMG from China reported that extended transsternal thymectomy is a safe and effective treatment with an estimated cumulative remission rate of $26.4 \%$ at 2 years after surgery (17). Of further note, Mineo et al. retrospectively reviewed 47 patients with OMG who underwent thymectomy and 62 patients with OMG who received only pharmacologic therapy, comparing the clinical outcomes between the two groups (18). In this study, Kaplan-Meier analysis showed estimated probabilities of remission at 5 years of $53 \%$ for the surgical group and $32 \%$ for the nonsurgical group.

Similarly, the estimated cumulative rates of CSR after thymectomy were $41.8 \%$ at 5 years for our whole series, and $48.3 \%$ at 5 years for the nonthymomatous OMG patients in our series. The ideal treatment is to alleviate symptoms with the least therapeutic risk. In this era of minimally invasive surgery, several minimally invasive approaches to thymectomy have been developed with satisfactory surgical and neurological outcomes (19-23). Minimally invasive thymectomy should be recommended to OMG patients with diplopia impairing quality of life and unsatisfactory response to adequate immunosuppressive medications.

Assessing prognostic factors plays a vital role in making treatment decisions and treating patients. In the analysis of factors influencing remission of OMG after surgery, data are not only limited, but also conflicting. A retrospective case series from China found that thymectomy within 1 year after disease onset was significantly associated with remission (17). Another retrospective study, including 135 patients with OMG, revealed that anti-titin antibody, thymus histopathology, and prednisone treatment were significant prognosticators (24). In an Italian study, Mineo et al. demonstrated that treatment within 6 months after disease onset was the only predictor of remission (18). Our results, however, suggest that age at disease onset of 40 years or younger is a significant predictor of remission after thymectomy. This discrepancy is possibly due to the diversity in the study population, the prognostic variables included in the analysis, the definition used for remission, and the type of statistical analyses used in the studies.

\section{Study limitations}

The main limitations of the study include the retrospective nature and small sample size with $11(17.7 \%)$ patients being lost to follow-up. Since patients who were lost to follow-up often have a different prognosis with those who were not (25), caution should be taken when interpreting the results of our study. In addition, this study was more likely to only include patients with severe symptoms and those who 
poorly respond to the pharmacological treatment. Despite these limitations, our retrospective study sheds light on the possibility of the identification of potential prognostic predictors for patients with OMG after thymectomy. Further multi-center studies with large sample sizes are needed to explore this highly relevant clinical topic.

\section{Conclusions}

Our results further confirmed the safety and efficacy of thymectomy in patients with OMG. CSR of OMG after thymectomy is likely to be achieved in about $40 \%$ of patients at 5 years and can be predicted by age at disease onset of 40 years or younger.

\section{Acknowledgments}

The authors greatly appreciate all individuals who participated in this study.

Funding: None.

\section{Footnote}

Conflicts of Interest: The authors have no conflicts of interest to declare.

Etbical Statement: The authors are accountable for all aspects of the work in ensuring that questions related to the accuracy or integrity of any part of the work are appropriately investigated and resolved. Ethics approval was granted by the ethics committee and institutional review board of the Second Affiliated Hospital of South China University. This study was conducted in accordance with the 1964 Helsinki Declaration and its amendments. Written informed consent of surgery and the use of clinical data were obtained from all patients involved in this study.

Open Access Statement: This is an Open Access article distributed in accordance with the Creative Commons Attribution-NonCommercial-NoDerivs 4.0 International License (CC BY-NC-ND 4.0), which permits the noncommercial replication and distribution of the article with the strict proviso that no changes or edits are made and the original work is properly cited (including links to both the formal publication through the relevant DOI and the license). See: https://creativecommons.org/licenses/by-nc-nd/4.0/.

\section{References}

1. Smith SV, Lee AG. Update on Ocular Myasthenia Gravis. Neurol Clin 2017;35:115-23.

2. Wong SH, Huda S, Vincent A, et al. Ocular Myasthenia Gravis: Controversies and Updates. Curr Neurol Neurosci Rep 2014;14:421.

3. Kerty E, Elsais A, Argov Z, et al. EFNS/ENS Guidelines for the treatment of ocular myasthenia. Eur J Neurol 2014;21:687-93.

4. Al-Haidar M, Benatar M, Kaminski HJ. Ocular Myasthenia. Neurol Clin 2018;36:241-51.

5. De Lott LB, Kerber KA, Lee PP, et al. DiplopiaRelated Ambulatory and Emergency Department Visits in the United States, 2003-2012. JAMA Ophthalmol 2017;135:1339-44.

6. Zhu K, Li JX, Huang X, et al. Thymectomy is a beneficial therapy for patients with non-thymomatous ocular myasthenia gravis: a systematic review and meta-analysis. Neurol Sci 2017;38:1753-60.

7. Gilhus NE. Myasthenia Gravis. N Engl J Med 2016;375:2570-81.

8. Wang LL, Zhang Y, He ML. Clinical Features and Prognosis of Ocular Myasthenia Gravis Patients with Different Phenotypes. Chin Med J (Engl) 2015;128:2682-4.

9. Kamarajah SK, Sadalage G, Palmer J, et al. Ocular presentation of myasthenia gravis: A natural history cohort. Muscle Nerve 2018;57:622-7.

10. Nagia L, Lemos J, Abusamra K, et al. Prognosis of Ocular Myasthenia Gravis: Retrospective Multicenter Analysis. Ophthalmology 2015;122:1517-21.

11. Sommer N, Sigg B, Melms A, et al. Ocular myasthenia gravis: response to long-term immunosuppressive treatment. J Neurol Neurosurg Psychiatry 1997;62:156-62.

12. Grob D. Course and management of myasthenia gravis. J Am Med Assoc 1953;153:529-32.

13. Lee YG, Kim US. Efficacy and Safety of Low-to-Moderate Dose Oral Corticosteroid Treatment in Ocular Myasthenia Gravis. J Pediatr Ophthalmol Strabismus 2018;55:339-42.

14. Wong SH, Petrie A, Plant GT. Ocular Myasthenia Gravis: Toward a Risk of Generalization Score and Sample Size Calculation for a Randomized Controlled Trial of Disease Modification. J Neuroophthalmol 2016;36:252-8.

15. Aguirre F, Villa AM. Prognosis of Ocular Myasthenia Gravis in an Argentinian Population. Eur Neurol 
2018;79:113-7.

16. Roberts PF, Venuta F, Rendina E, et al. Thymectomy in the treatment of ocular myasthenia gravis. J Thorac Cardiovasc Surg 2001;122:562-8.

17. Liu Z, Feng H, Yeung SC, et al. Extended transsternal thymectomy for the treatment of ocular myasthenia gravis. Ann Thorac Surg 2011;92:1993-9.

18. Mineo TC, Ambrogi V. Outcomes after thymectomy in class I myasthenia gravis. J Thorac Cardiovasc Surg 2013;145:1319-24.

19. Ruckert JC, Ismail M, Swierzy M, et al. Thoracoscopic thymectomy with the da Vinci robotic system for myasthenia gravis. Ann N Y Acad Sci 2008;1132:329-35.

20. Zielinski M, Hauer L, Hauer J, et al. Comparison of complete remission rates after 5 year follow-up of three different techniques of thymectomy for myasthenia gravis.
Eur J Cardiothorac Surg 2010;37:1137-43.

21. Tomulescu V, Sgarbura O, Stanescu C, et al. TenYear Results of Thoracoscopic Unilateral Extended Thymectomy Performed in Nonthymomatous Myasthenia Gravis. Ann Surg 2011;254:761-6.

22. Novellino L, Longoni M, Spinelli L, et al. "Extended" thymectomy, without sternotomy, performed by cervicotomy and thoracoscopic technique in the treatment of myasthenia gravis. Int Surg 1994;79:378-81.

23. Singh G, Glotzbach J, Costa J, et al. Minimally invasive radical thymectomy. Ann Cardiothorac Surg 2016;5:59-61.

24. Deng Y, Zhang X. Prognosis of thymectomy in treatment of ocular myasthenia gravis and relevant influencing factors. Zhonghua Yi Xue Za Zhi 2009;89:1922-5.

25. Dettori JR. Loss to follow-up. Evid Based Spine Care J 2011;2:7-10.
Cite this article as: Liu X, Zhou W, Hu J, Hu M, Gao W, Zhang S, Zeng W. Prognostic predictors of remission in ocular myasthenia after thymectomy. J Thorac Dis 2020;12(3):422-430. doi: $10.21037 /$ jtd.2020.01.17 